\title{
Scattering of an obliquely incident plane wave from a circular clad rod
}

\author{
F. Honarvar and A. N. Sinclair \\ Department of Mechanical and Industrial Engineering, University of Toronto, 5 King's College Road, \\ Toronto, Ontario M5S 3G8, Canada
}

(Received 12 September 1996; accepted for publication 11 February 1997)

\begin{abstract}
The problem of scattering of an obliquely incident plane acoustic wave from an infinite solid elastic clad rod is formulated. Numerical calculations show the effects of the variations of the cladding thickness on both the backscattered pressure spectrum and individual normal modes. It is shown that various resonance frequencies have different levels of sensitivity to variations of the cladding thickness. Experimental measurements are also performed at both normal and oblique incident angles on a copper-clad aluminum rod; good agreement is observed between the calculated and the measured results. These results demonstrate the potential of acoustic resonance scattering procedures for application in nondestructive evaluation (NDE) of clad rods. (C) 1997 Acoustical Society of America. [S0001-4966(97)01907-3]
\end{abstract}

PACS numbers: 43.20.Bi, 43.20.Fn, 43.20.Ks [ANN]

\section{INTRODUCTION}

There has been significant progress in both experimental and numerical studies of the scatter of acoustic waves from spheres and cylinders. There exists a potential for using these techniques in the field of nondestructive evaluation (NDE) of materials. This applies particularly to the NDE of long cylindrical components (such as rods, wires, tubes, etc.) which fall into the class of the few geometries for which the scattered pressure field has an explicit solution in the form of an infinite series. While studies on acoustic wave scattering from circular cylinders and shells are abundant in the literature, very little has been investigated about acoustic wave scattering from multi-layered cylinders. Particularly, the case of a plane acoustic wave obliquely incident on a two-layered cylinder (clad rod) has not been studied before. The study of the obliquely incident wave is important because, in practice, it is impossible to have a perfect normally incident plane wave, and traces of the obliquely incident components of the wave always show up in the backscattered pressure spectrum.

The physics of the scattering of an acoustic wave from an elastic target is a complicated phenomenon that involves the generation of different types of surface waves outside and inside the elastic target. Whenever an acoustic wave strikes the boundary of a cylinder, a specular reflection is returned. Waves are also launched from selected points on that boundary that circumnavigate the body in spiral or helical trajectories. These surface waves propagate near the boundary, either in the outer medium, or in the body of the scatterer. ${ }^{1}$ The waves that propagate on the fluid side of the fluid-solid interface are Franz waves and Scholte-Stoneley waves (also called creeping waves) ${ }^{2-4}$ When the cylinder is insonified by a normally incident plane acoustic wave, two distinct families of waves are generated inside the elastic cylinder. The first group are the Rayleigh-type (or more correctly pseudo-Rayleigh-type) waves and the other group are the whispering gallery-type (WG) waves. ${ }^{1}$ It has been established that a close relationship exists between the resonances of an elastic scatterer and the circumferential waves which propagate along its periphery. ${ }^{5}$ These surface waves and their relationship with the natural resonances of the scatterer have been extensively studied for the case of elastic cylinders. If the frequency of the incident wave coincides with one of the vibrational eigenfrequencies of the cylinder, surface waves match phases upon circumnavigation so as to build up to the resonance by constructive interference. When the wave is obliquely incident on the cylinder, additional types of waves are generated in the cylinder, and correspondingly additional resonance modes of the cylinder are excited. These additional (surface) waves are called guided waves (meaning axially guided waves $)^{6-8}$ and are mostly of a transverse nature. In the case of an obliquely incident wave, the travel path of all surface waves are helices. The angle of each helix depends on the phase velocity of the corresponding surface wave. $^{9}$

Analytical and numerical studies of acoustic wave scattering from cylinders were initiated by Faran in 1951. ${ }^{10}$ Resonance scattering theory (RST) ${ }^{11}$ was one of the principal theoretical works in this area which presented a physical interpretation of the resonances generated in the body of the scatterer due to the constructive interference of the surface waves. RST also showed that the frequency spectrum of the backscattered echo is composed of a resonant part superimposed on a nonresonant spectrum, and that these two parts can be separated analytically. Flax et al. ${ }^{12}$ formulated the problem of scattering of an obliquely incident plane wave from an infinite elastic cylinder. Surface waves generated by a plane acoustic wave obliquely incident on a cylinder were studied by Nagl et al. ${ }^{9}$ based on RST and the Watson transformation. Veksler ${ }^{13}$ computed the modal resonances for an aluminum cylinder insonified by an obliquely incident wave and showed their correspondence to the extrema of the backscattered spectrum. A geometrical interpretation of the phase matching of the helical surface waves was given by Conoir et al. ${ }^{14}$ who also showed that the resonances shift toward higher frequencies when the angle of incidence is increased. 


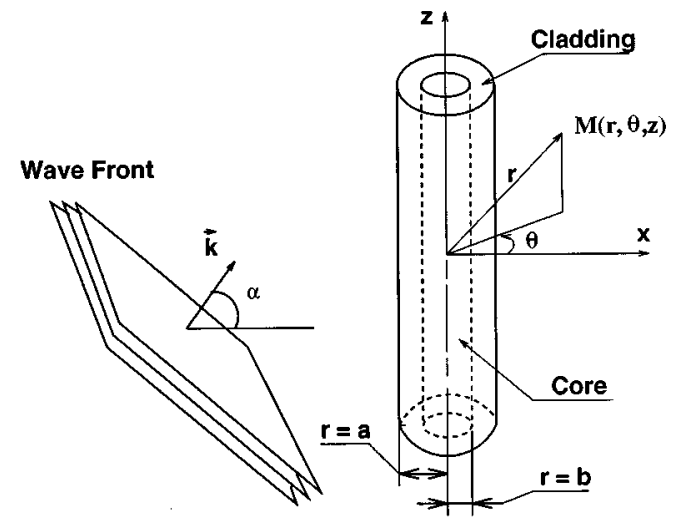

FIG. 1. Geometry of a plane wave obliquely incident on a submerged clad rod.

The major thrust in experimental work was made by the advent of the Method of Isolation and Identification of Resonances (MIIR) developed by French acousticians. ${ }^{6,15}$ Using MIIR, the scattered spectrum, the resonance spectrum, and the mode shape of each resonance can be experimentally measured. Although tone bursts of long duration were used in early MIIR studies, it was later shown that short pulses can provide the same information. ${ }^{16,17}$

The problem of acoustic wave scattering from multilayered cylinders has also been considered by a few researchers. Flax and Neubauer ${ }^{18}$ did the theoretical formulation and numerical evaluation of acoustic wave scattering from isotropic two-layered absorptive cylindrical shells. Sinclair and Addison ${ }^{19}$ developed the equations of the scattering of a plane acoustic/elastic wave from a two-layered cylinder embedded in a solid or a fluid medium. The scattering of ultrasonic waves by two concentric fluid cylinders was investigated by Sinai and $\mathrm{Wagg}^{20}$ and acoustic scattering from two eccentric cylinders was studied by Roumeliotis et al. ${ }^{21}$ However, all of these works only consider the case of normally incident waves.

In this paper the detailed formulation for the scattering of an obliquely incident plane acoustic wave from a submerged clad rod is developed and the backscattered pressure spectrum of clad rods with various cladding thickness ratios are numerically evaluated. Experimental results, corresponding to small angles of incidence, are used to verify the mathematical calculations.

\section{THEORY}

Figure 1 shows an infinite plane acoustic wave of frequency $\omega / 2 \pi$ incident at an angle $\alpha$ on a submerged clad rod of infinite length, outer radius $a$, and core radius $b$. A cylindrical coordinate system $(r, \theta, z)$ is chosen with the $z$ direction coincident with the axis of the cylinder. The pressure of the incident plane wave $p_{i}$ at time $t$, external to the cylinder at an arbitrary point $M(r, \theta, z)$ is represented by, ${ }^{12}$

$$
p_{i}=p \sum_{0}^{\infty} \varepsilon_{n=0} i^{n} J_{n}\left(k_{\perp} r\right) \cos (n \theta) e^{i\left(k_{z} z-\omega t\right)},
$$

where

$$
k_{z}=k \sin \alpha, \quad k_{\perp}=k \cos \alpha,
$$

and $k=\omega / c, c$ is the compressional wave velocity in the fluid medium outside the cylinder, $\varepsilon_{n}$ is the Neumann factor $\left(\varepsilon_{n}=2-\delta_{n 0}\right), p_{0}$ is the incident pressure wave amplitude, and $J_{n}$ are the Bessel functions of the first kind of order $n$. The outgoing scattered wave pressure $p_{s}$ at point $M$ must be symmetrical about $\theta=0$ and, therefore, of the form,

$$
p_{s}=p \sum_{0} \sum_{n=0}^{\infty} \varepsilon_{n} i^{n} A_{n} H_{n}^{(1)}\left(k_{\perp} r\right) \cos (n \theta) e^{i\left(k_{z} z-\omega t\right)},
$$

where $H_{n}^{(1)}$ are the Hankel functions of the first kind of order $n$, and $A_{n}$ are the unknown scattering coefficients. If the isotropic core and cladding media are designated by subscript $i=1,2$, respectively, then using Helmholtz displacement potential functions, $\phi_{i}$ (scalar) and $\psi_{i}$ (vector), the Navier's equation for each medium can be written as

$$
\begin{aligned}
& \boldsymbol{\nabla}\left(\left(\lambda_{i}+2 \mu_{i}\right) \nabla^{2} \phi_{i}-\rho_{i} \frac{\partial^{2} \phi_{i}}{\partial t^{2}}\right)+\nabla \times\left(\mu_{i} \nabla^{2} \boldsymbol{\psi}_{i}-\rho_{i} \frac{\partial^{2} \boldsymbol{\psi}_{i}}{\partial t^{2}}\right) \\
& \quad=0, \quad i=1,2,
\end{aligned}
$$

where $\lambda_{i}$ and $\mu_{i}$ are the Lamé constants and $\rho_{i}$ are the material densities. By expanding Eq. (4), four partial differential equations in terms of potential functions are obtained for each medium. In order to satisfy these partial differential equations, the potential functions should be of the following forms:

$$
\begin{aligned}
& \phi_{1}=\sum_{n=0}^{\infty} B_{n} J_{n}\left(k_{L_{1}} r\right) \cos (n \theta) e^{i\left(k_{z} z-\omega t\right)}, \\
& {\left[\psi_{1}\right]_{r}=\sum_{n=0}^{\infty} C_{n} J_{n+1}\left(k_{T_{1}} r\right) \sin (n \theta) e^{i\left(k_{z} z^{-\omega t)}\right.},} \\
& {\left[\psi_{1}\right]_{\theta}=\sum_{n=0}^{\infty}-C_{n} J_{n+1}\left(k_{T_{1}} r\right) \cos (n \theta) e^{i\left(k_{z} z-\omega t\right)},} \\
& {\left[\psi_{1}\right]_{z}=\sum_{n=0}^{\infty} D_{n} J_{n}\left(k_{T_{1}} r\right) \sin (n \theta) e^{i\left(k_{z} z-\omega t\right)},} \\
& \phi_{2}=\sum_{n=0}^{\infty}\left[E_{n} J_{n}\left(k_{L_{2}} r\right)+F_{n} Y_{n}\left(k_{L_{2}} r\right)\right] \cos (n \theta) e^{i\left(k_{z} z-\omega t\right)} \text {, } \\
& {\left[\psi_{2}\right]_{r}=\sum_{n=0}^{\infty}\left[K_{n} J_{n+1}\left(k_{T_{2}} r\right)\right.} \\
& \left.+L_{n} Y_{n+1}\left(k_{T_{2}} r\right)\right] \sin (n \theta) e^{i\left(k_{z} z-\omega t\right)}, \\
& {\left[\psi_{2}\right]_{\theta}=\sum_{n=0}^{\infty}\left[-K_{n+1} J_{n}\left(k_{T_{2}} r\right)\right.} \\
& \left.-L_{n} Y_{n+1}\left(k_{T_{2}} r\right)\right] \cos (n \theta) e^{i\left(k_{z} z-\omega t\right)}, \\
& {\left[\psi_{2}\right]_{z}=\sum_{n=0}^{\infty}\left[M_{n} J_{n}\left(k_{T_{2}} r\right)\right.} \\
& \left.+N_{n} Y_{n}\left(k_{T_{2}} r\right)\right] \sin (n \theta) e^{i\left(k_{z} z-\omega t\right)}
\end{aligned}
$$


where

$$
k_{L_{i}}=\left(\frac{\omega}{c_{L_{i}}}\right)^{2}-k_{z}^{2}, \quad k_{T_{i}}=\left(\frac{\omega}{c_{T_{i}}}\right)^{2}-k_{z}^{2}, \quad i=1,2 .
$$

$c_{L_{i}}$ and $c_{T_{i}}$ are the compressional and shear wave velocities respectively and $B_{n}, C_{n}, \ldots$ are unknown coefficients. By applying the boundary conditions at the core-cladding and fluid-cladding interfaces to Eqs. (5), the following system of linear algebraic equations is obtained,

$$
\left[D_{n}^{(1)}\right]\left\{\mathscr{C}_{n}\right\}=\left\{\mathscr{B}_{n}\right\},
$$

where

$\left\{\mathscr{C}_{n}\right\}=\left[\begin{array}{llllllll}A_{n} & B_{n} C_{n} & D_{n} & E_{n} & F_{n} & K_{n} & L_{n} & M_{n} N_{n}\end{array}\right]^{T}$,

$\left\{\mathscr{B}_{n}\right\}=\left[\begin{array}{llllllllll}b_{1} & b_{2} & 0 & 0 & 0 & 0 & 0 & 0 & 0 & 0\end{array}\right]^{T}$,

and $D_{n}^{(1)}$ is a $10 \times 10$ matrix. ${ }^{22}$ Equation (7) could be solved for $A_{n}$ at any given value of normalized frequency $k a$ using Cramer's rule.

The scattered pressure field is usually evaluated in the far-field $(r \gg a)$ at a fixed angle $\theta$ for a range of frequencies. The resulting normalized far-field amplitude spectrum, which is called the form function, is obtained from the following equation, ${ }^{23}$

$$
f_{\infty}(\theta, k a)=\left(\frac{2 r}{a}\right)^{1 / 2}\left(\frac{p_{s}}{p_{0}}\right) e^{-i k_{\perp} r} .
$$

The form function can be decomposed into a sum of normal modes,

$$
f_{\infty}(\theta, k a)=\sum_{n=0}^{\infty} f_{n}(\theta, k a),
$$

where the individual normal modes are defined as:

$$
f_{n}(\theta, k a)=\frac{2}{\sqrt{i \pi k_{\perp} a}} \varepsilon_{n} A_{n} \cos (n \theta) .
$$

For a cylindrical geometry, the nonresonant background scattering component (rigid background here) can be sepa-
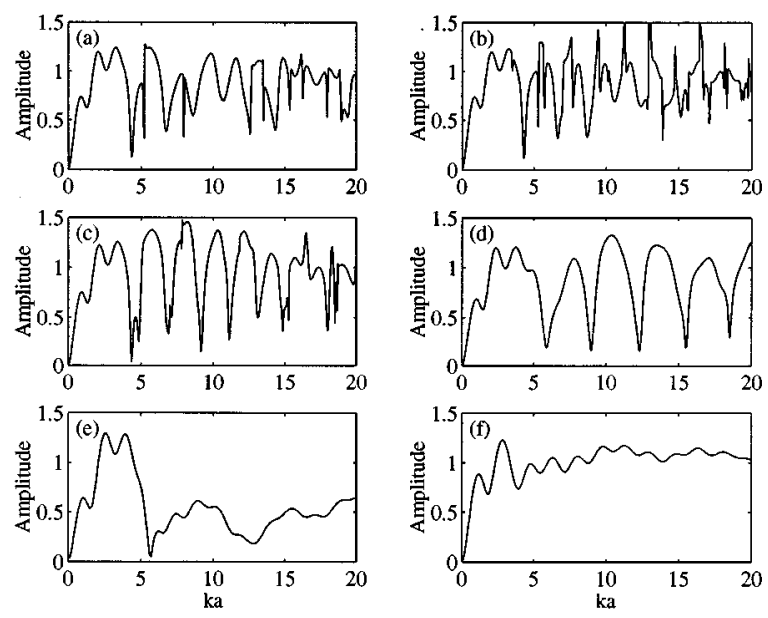

FIG. 2. Calculated form function of a copper-clad aluminum rod: (a) $\alpha=0^{\circ}$; (b) $\alpha=5^{\circ}$; (c) $\alpha=15^{\circ}$; (d) $\alpha=30^{\circ}$; (e) $\alpha=37^{\circ}$; (f) $\alpha=45^{\circ}$. rated from the resonant part. For a rigid cylinder the scattering coefficients $A_{n}^{\text {(rigid) }}$ are given as: ${ }^{23}$

$$
A_{n}^{(\text {rigid) }}(k a)=-\frac{J_{n}{ }^{\prime}(k a)}{H_{n}^{(1)^{\prime}}(k a)} .
$$

The resonant part of each mode can be obtained by subtracting the modal $(n=0,1,2, \ldots)$ components of the rigid background $f_{n}^{(\text {rigid) }}(\theta, k a)$ from each of the normal mode components $f_{n}(\theta, k a)$ of the form function $f_{\infty}(\theta, k a)$ according to the following equation:

$$
f_{n}^{(\mathrm{res})}(\theta, k a)=\frac{2}{\sqrt{i \pi k_{\perp} a}} \varepsilon_{n}\left[A_{n}-A_{n}^{(\mathrm{rigid})}\right] \cos (n \theta) .
$$

\section{EVALUATION OF THE FORM FUNCTION AND RESONANCE SPECTRUM}

Form functions of a copper-clad aluminum rod are calculated for various incident angles of the plane wave, see Fig. 2. Physical properties of the cladding and the core materials are given in Table I. The outer diameter of the clad rod is $a=9.15 \mathrm{~mm}$ and its core diameter is $b=8.70 \mathrm{~mm}$. Similar to the case of an isotropic elastic cylinder, ${ }^{12,14}$ when the incident angle is increased from $\alpha=0^{\circ}$, initially new minima corresponding to the resonances associated with guided waves are observed on the form function, see Fig. 2(b). With further increase of $\alpha$, all resonances (minima on the curve) shift to higher frequencies. Beyond the second critical angle, $\alpha=41.42^{\circ}$, no elastic resonance can be detected on the form function and the form function becomes similar to that of a rigid cylinder.

The calculated form functions for a number of copperclad aluminum rods with different cladding thickness ratios are shown in Fig. 3. The form functions are evaluated for $\alpha=3^{\circ}$ and $0 \leqslant k a \leqslant 20$. The cladding thickness ratio is defined as:

$$
h=\frac{a-b}{a} .
$$

Therefore $h=0$ refers to an aluminum cylinder with no cladding and $h=1$ is a simple copper cylinder. The common notation of designating the resonant modes by two indices $(n, l)$ is used, where $n$ is the mode number and $l$ is the eigenfrequency label. $l=1$ corresponds to a Rayleigh-type wave and $l=2,3, \ldots$ corresponds to whispering gallery-type waves. Guided waves are designated as $\langle n, p\rangle$ where $n$ is the mode number and $p$ is the eigenfrequency label of the resonance.

From Fig. 3 it can be seen that with the increase of the cladding thickness from zero, the form function starts deviating from that of an aluminum cylinder $(h=0)$ and gradu-

TABLE I. Physical properties of aluminum, copper, and tungsten. ${ }^{22}$

\begin{tabular}{lccc}
\hline \hline & $\begin{array}{c}\rho \\
\left(\mathrm{kg} / \mathrm{m}^{3}\right)\end{array}$ & $\begin{array}{c}c_{L} \\
(\mathrm{~m} / \mathrm{s})\end{array}$ & $\begin{array}{c}c_{T} \\
(\mathrm{~m} / \mathrm{s})\end{array}$ \\
\hline Aluminum & 2694 & 6427 & 3112 \\
Copper & 8900 & 4600 & 2160 \\
Tungsten & 19100 & 5460 & 2620 \\
\hline \hline
\end{tabular}

F. Honarvar and A. N. Sinclair: Scattering from clad rods 


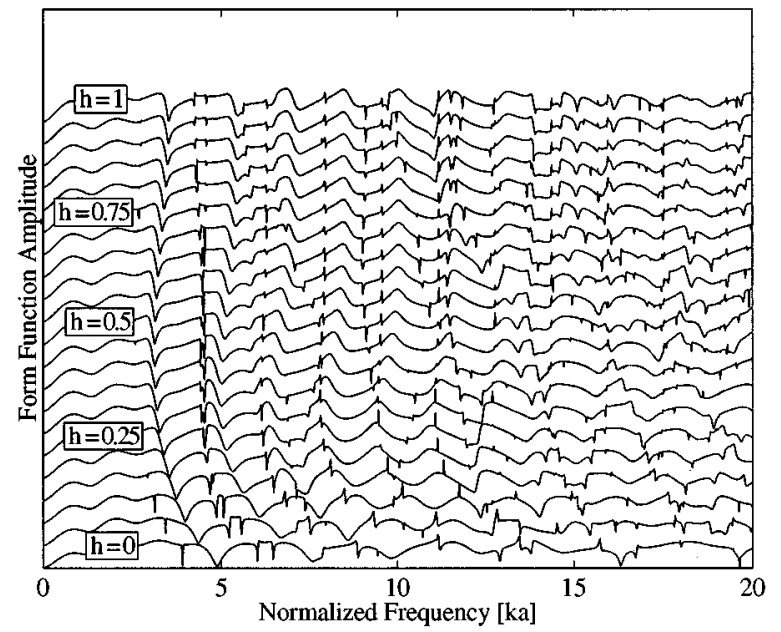

FIG. 3. Variation of the form function of the copper-clad aluminum rod with changes in cladding thickness at $\alpha=3^{\circ}$.

ally approaches the form function of a simple copper cylinder $(h=1)$. It can be observed that the resonance frequencies of the clad rod are significantly dependent on the thickness of the cladding.

Figure 4 shows the resonance spectra of the first three normal modes of the clad rod. These curves are evaluated from Eq. (14). The dependence of the resonance frequencies on cladding thickness can be observed more clearly with these resonance spectra than on the form functions of Fig. 3. Both the frequency and the quality of the resonances which are visible in these spectra vary with changes in $h$. Not many of the breathing mode $(n=0)$ eigenfrequencies are excited in the frequency range considered and many of these resonances are so weak that they do not appear in the spectrum. The first two eigenfrequencies of the dipole mode $(n=1)$ corresponding to the whispering gallery-type wave $(1,2)$ and guided wave $\langle 1,1\rangle$ remain almost constant in the range $0.25 \leqslant h \leqslant 1$. The first eigenfrequency of the Rayleigh-type wave $(2,1)$ and guided wave $\langle 2,1\rangle$ of the quadrupole mode show a similar behavior for the range $0.5 \leqslant h \leqslant 1$. This indicates that the surface waves corresponding to these eigenfrequencies do not penetrate deeply into the material and mostly propagate on the surface.

Resonances corresponding to higher values of $l$ for all three modes show higher sensitivity to variations of the cladding thickness. This clearly indicates that high frequency resonances can be good indicators of the cladding thickness. The variations of the frequencies of these resonances with an increase in the value of $h$ is not necessarily monotonic. This behavior can be correlated to the radial nodes of these resonances. ${ }^{24}$ For a given mode number $n$, this nonmonotonic dependence of resonance frequencies on $h$ becomes more severe with the increase of the eigenfrequency label $l$ which corresponds to an increase in the number of radial nodes of the surface wave associated with that resonance.

It can also be shown that the nonmonotonic behavior of each resonance frequency with variations of $h$ is consistent with the dependence of phase velocities of surface waves on $h$. The phase velocity of a helical surface wave is defined as ${ }^{9}$
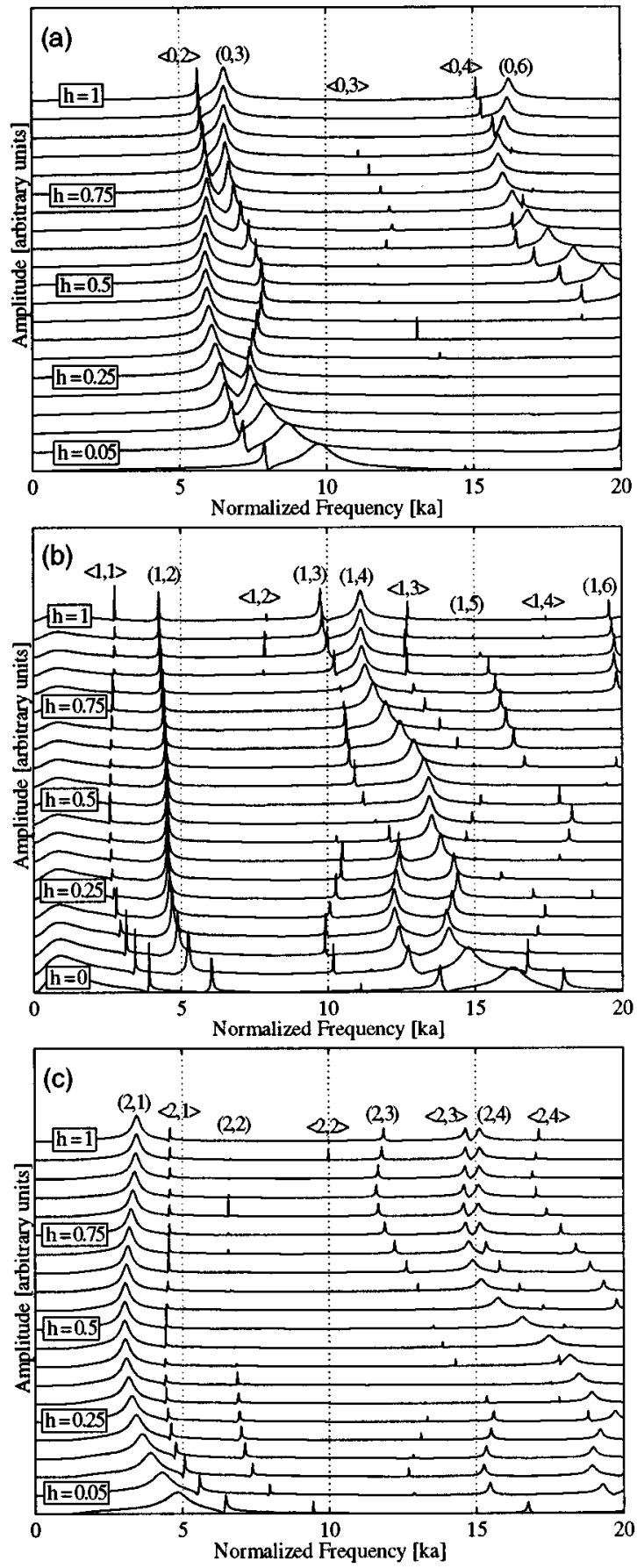

FIG. 4. Variation of the resonance spectra of the copper-clad aluminum rod with changes in cladding thickness at $\alpha=3^{\circ}$ : (a) breathing mode $(n=0)$; (b) dipole mode $(n=1)$; (c) quadrupole mode $(n=2)$.

$$
c_{l}^{p h}=c\left\{\left(n_{l} / k a\right)^{2}+\sin ^{2} \alpha\right\}^{-1 / 2},
$$

where $n_{l}$ is the mode number of the resonance and $k a$ is its frequency. In Fig. 5(a) and (b), the phase velocities of the surface waves shown in Fig. 4(b) and (c) are plotted as a function of the cladding thickness ratio $h$. The behavior of the phase velocities of these surface waves is similar to that of their corresponding resonance frequencies in Fig. 4(b) and (c). This indicates that the primary reason for the nonmonotonic behavior observed in Fig. 4(a), (b), and (c) is the changes in the phase velocities of these surface waves. 

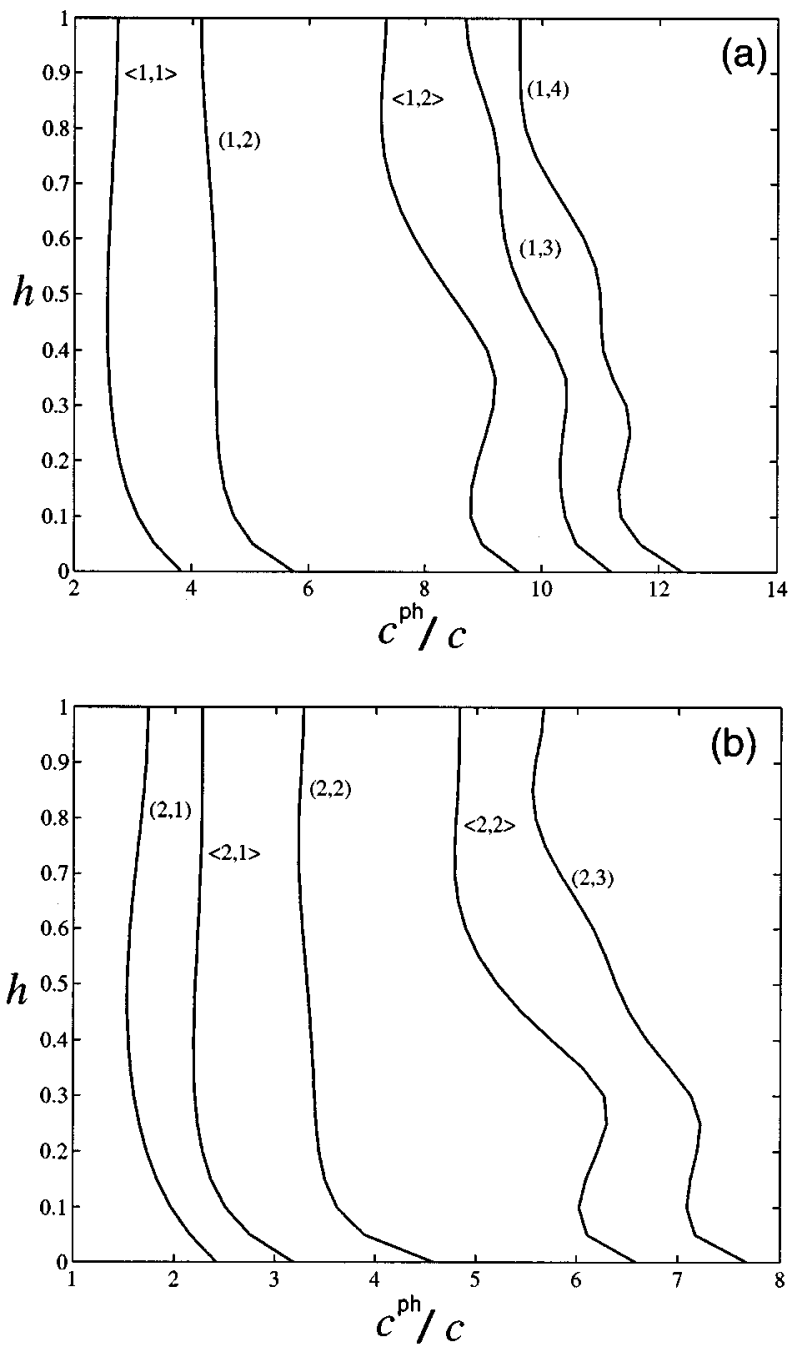

FIG. 5. Variation of the phase velocities of various surface waves with changes in cladding thickness of the copper clad aluminum rod: (a) dipole mode $(n=1)$; (b) quadrupole mode $(n=2)$.

Changes in the phase velocities of the surface waves are in turn due to the difference in the bulk wave velocities of the core and the cladding media.

\section{EXPERIMENTS}

\section{A. Measurement of the backscattered pressure spectrum}

A schematic diagram of the experimental setup for measuring the backscattered pressure field and the resonance spectrum using short pulses is shown in Fig. 6. The sample tested was a copper-clad aluminum rod. Physical properties of the cladding and the core metals are given in Table I and correspond to the numerical calculations of Section II. The velocity of sound in water was calculated from the water temperature according to the following equation, ${ }^{25}$

$$
c_{w}=\sum_{n=0}^{5} g_{n} T^{n},
$$

where the coefficients $g_{n}$ are listed in Table 3.3. of Reference 25 and $T$ is the water temperature in ${ }^{\circ} \mathrm{C}$. Two identical broadband ultrasonic transducers were used as transmitter

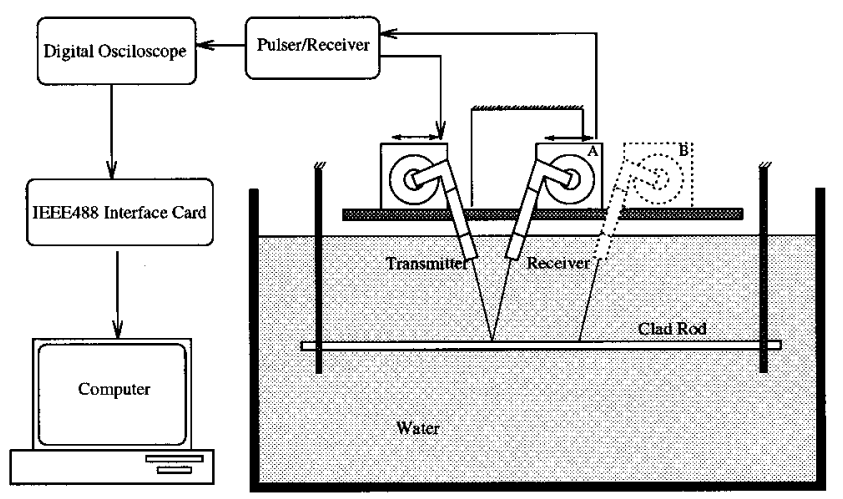

FIG. 6. Experimental setup for measuring the backscattered spectrum.

and receiver. Each transducer had a diameter of $1.125 \mathrm{in.}$ and a nominal center frequency of $500 \mathrm{kHz}$. The useful frequency range of each transducer spanned from approximately 200 to $800 \mathrm{kHz}$. In all the experiments, the sampling frequency was $50 \mathrm{MHz}$. For the clad rod, $a=9.15 \mathrm{~mm}$ and $b=8.70 \mathrm{~mm}$, and the length of the rod was $350 \mathrm{~mm}$.

Frequency characteristics of the transmitting and receiving transducers were compensated by a deconvolution technique according to Reference 26. For this purpose a 0.25 mm-diam tungsten fiber was used to obtain a reference spectrum. Since the $k a$ values for the tungsten fiber in the aforementioned frequency range are very small, none of the resonance frequencies of the tungsten fiber were excited and its frequency spectrum was smooth, see Fig. 7.

The frequency spectrum of the measured signal $S(\omega)$ for the copper-clad aluminum rod is a convolution of the cylinder transfer function $p_{s}(\omega) / p_{0}(\omega)$, and the frequency characteristics of the experimental system $G(\omega)$, i.e.,

$$
|S(\omega)|=\left|G(\omega) \frac{p_{s}(\omega)}{p_{0}(\omega)}\right| .
$$

A similar relationship exists for the tungsten fiber

$$
\left|S^{\prime}(\omega)\right|=\left|G(\omega) \frac{p_{s}^{\prime}(\omega)}{p_{0}(\omega)}\right|,
$$

where $S^{\prime}(\omega)$ is the frequency spectrum of the measured signal and $p_{s}^{\prime}(\omega)$ is the scattered pressure field for the tungsten fiber. The form function of the tungsten fiber is calculated from the following equation:

$$
\left|f_{\infty}^{\prime}\right|=\left(\frac{2 r^{\prime}}{a^{\prime}}\right)^{1 / 2}\left|\frac{p_{s}^{\prime}(\omega)}{p_{0}(\omega)}\right| e^{-i k r^{\prime} \cos \alpha^{\prime}} .
$$

By substituting Eq. (20) into Eq. (19), one obtains

$$
|G(\omega)|=\left|\frac{S^{\prime}(\omega)}{f_{\infty}^{\prime}}\right|\left(\frac{2 r^{\prime}}{a^{\prime}}\right)^{1 / 2} e^{-i k r^{\prime} \cos \alpha^{\prime}} .
$$

Moreover, substituting Eq. (10) into Eq. (18) results in 

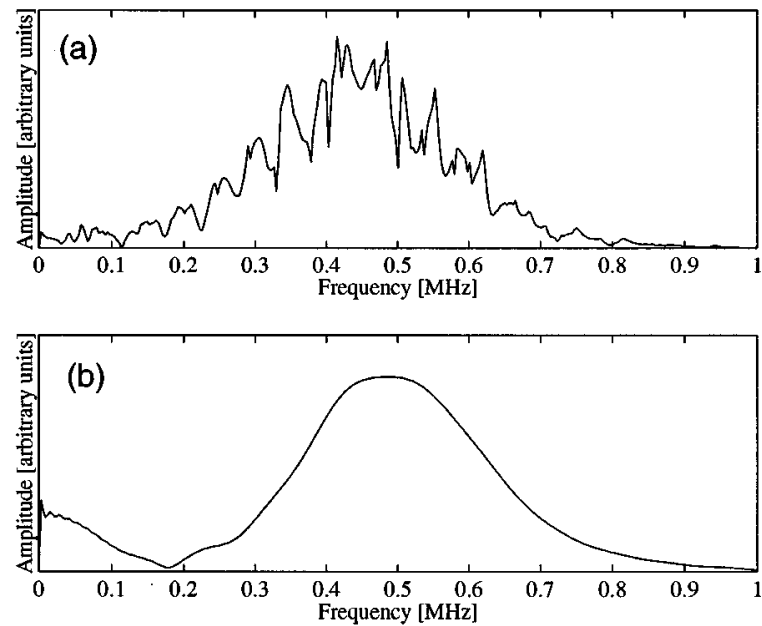

FIG. 7. Experimental frequency spectra of (a) copper-clad aluminum rod, (b) tungsten fiber.

$$
|G(\omega)|=\left|\frac{S(\omega)}{f_{\infty}}\right|\left(\frac{2 r}{a}\right)^{1 / 2} e^{-i k r \cos \alpha} .
$$

Equating the right-hand sides of Eqs. (21) and (22) leads to the following equation:

$$
\left|f_{\infty}\right|=\left|\frac{S(\omega)}{S^{\prime}(\omega)}\right|\left(\frac{a^{\prime} r}{a r^{\prime}}\right)^{1 / 2} e^{i k\left(r^{\prime} \cos \alpha^{\prime}-r \cos \alpha\right)}\left|f_{\infty}^{\prime}\right| .
$$

For the case of Reference 26 where $r^{\prime}=r \cos \alpha$ and $\alpha^{\prime}=0$, Eq. (23) reduces to the simpler expression of Eq. 27 of that paper. In Eq. (23) $S(\omega)$ and $S^{\prime}(\omega)$ are measured experimentally and $\left|f_{\infty}^{\prime}\right|$ is calculated numerically. The elastic properties of tungsten were obtained from Ref. 27 (Table A.1) see Table I. By measuring the backscattered echos from the sample rod and the tungsten fiber under similar conditions, one can obtain the form function of the rod from Eq. (23). Preliminary tests indicated that the best correspondence between measured and calculated form functions is obtained when $\alpha=\alpha^{\prime}$.
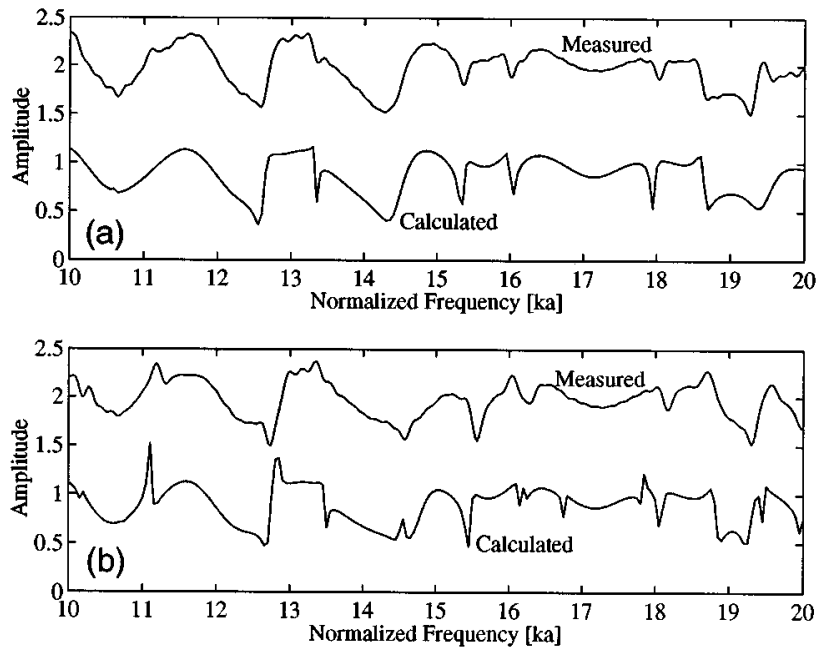

FIG. 8. Measured and calculated form functions for the copper-clad aluminum rod. (a) $\alpha=0$ (normal incidence); (b) $\alpha=3^{\circ}$.
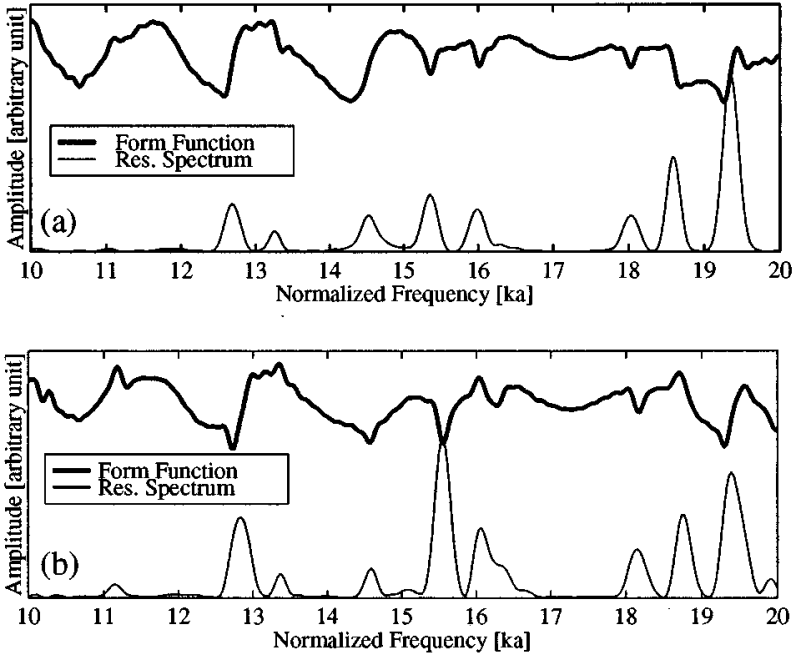

FIG. 9. Measured form functions and resonance spectra for the copper-clad aluminum rod. (a) $\alpha=0$ (normal incidence); (b) $\alpha=3^{\circ}$.

It is impossible to insonify the cylinder at a normal angle with a truly infinite plane wave. General purpose NDE transducers cannot produce a completely normally incident wave because even when the surface of the transducer is exactly parallel to the axis of the cylinder (in a pulse-echo arrangement), some oblique components will still appear in the signal because of the nonplanar characteristics of the incident wave. The smaller the diameter of the receiver and the longer the distance between the probe and the cylinder, the less will be the effects of oblique components. In practice, there are limitations on the diameter of the probe. The maximum distance between the probe and the sample also depends on the energy of the signal and dimensions of the water tank. Moreover, the directivity pattern of a transducer restricts the measurements to small incident angles. ${ }^{8,26}$

Figure 8 shows the measured and calculated form functions for the clad rod sample. The numerically calculated and experimentally measured form functions are found to be consistent for both normally and obliquely incident waves. Figure 9 shows the measured form functions and the resonance
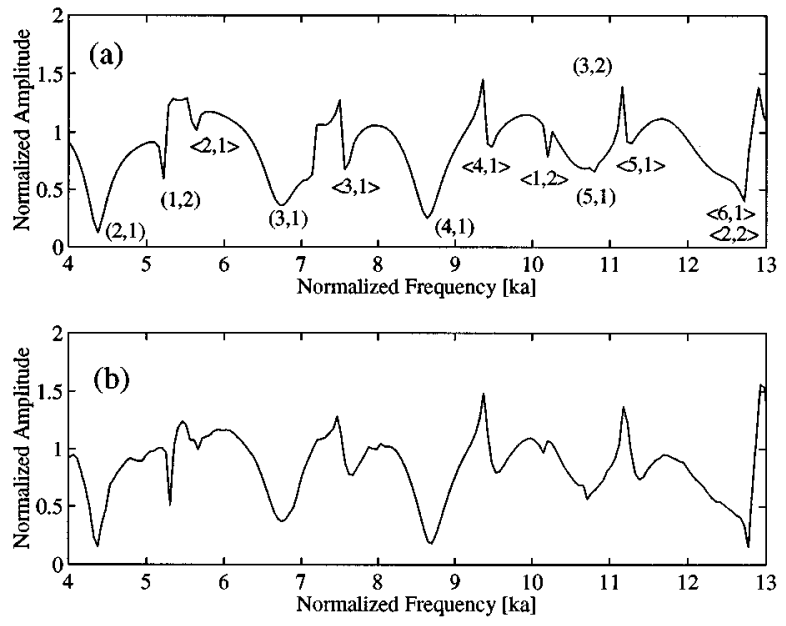

FIG. 10. Form functions for $8.026 \mathrm{~mm}$ diameter copper-clad aluminum rod at $\alpha=3^{\circ}$. (a) Calculated. (b) Measured.

F. Honarvar and A. N. Sinclair: Scattering from clad rods 


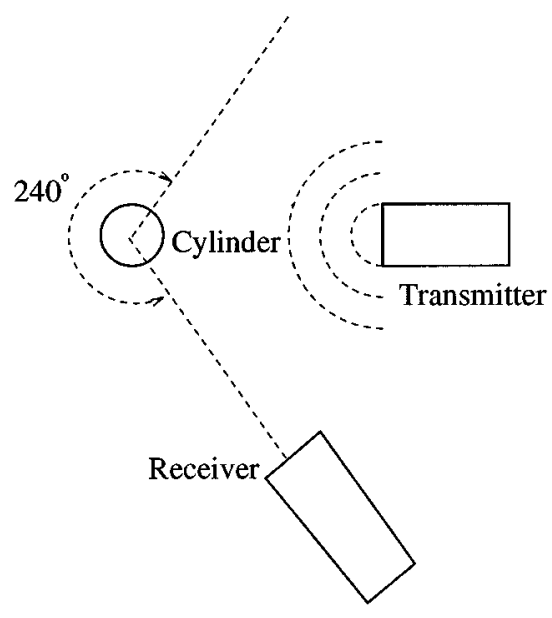

FIG. 11. Schematic diagram of the setup used for measuring the mode shape of the resonances.

spectra for the clad rod. The resonance spectra were obtained by removing the specular echo from the signal and then finding the power spectrum of the remaining echo. From these figures, it can be observed that when the cylinder is insonified at small oblique angles, the majority of the resonances

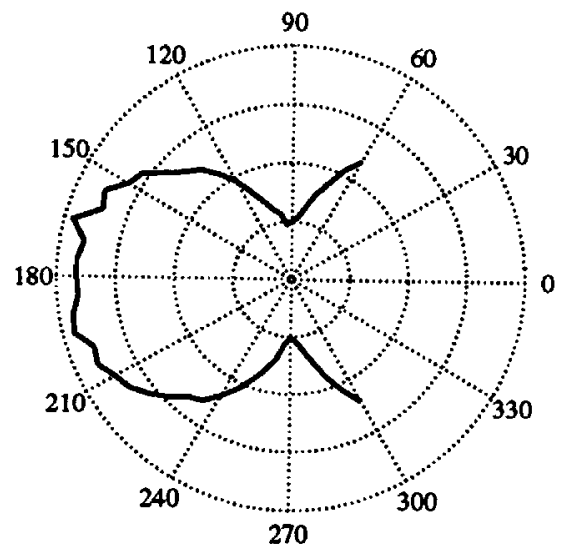

$(1,2)$

$\mathrm{ka}=\mathbf{5 . 3}$

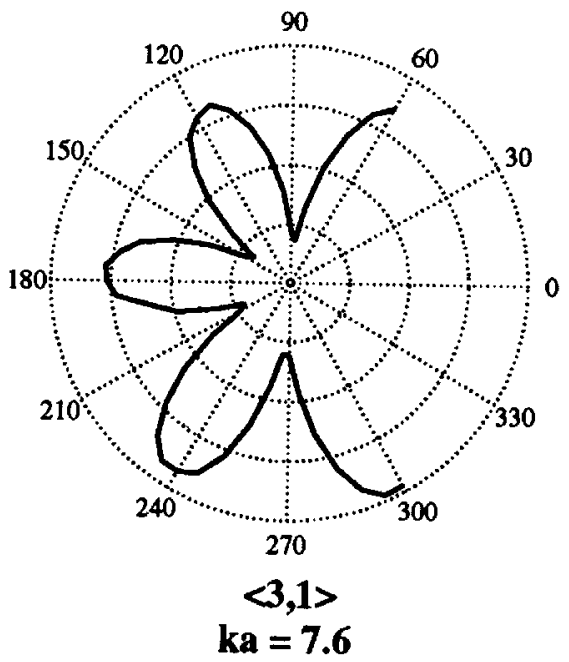

that were visible at normal incidence are shifted to the left and additional resonances appear in the spectrum which are due to the axially guided waves. There is good agreement between experimental measurements and theoretical calculations.

Faint evidence of axially guided mode resonances (e.g., at $k a \approx 11$ ) can be observed in Figs. 8(a) and 9(a), corresponding to nominally zero degrees angle of incidence. This is due to the nonplanar characteristics of the incident wave as mentioned earlier.

\section{B. Characterization of individual modes}

Using short pulse MIIR, ${ }^{17}$ the mode shapes of several resonances were measured at both normally and obliquely incident angles. The measurements were performed on a copper-clad aluminum rod with $2 a=8.026 \mathrm{~mm}$ and $h=0.05$. The frequency range for this sample was $4 \leqslant k a \leqslant 13$. The calculated and measured form functions for this sample are shown in Fig. 10.

Figure 11 shows the schematic diagram of the setup used for measuring the mode shapes. The transmitting transducer can insonify the cylinder at both normally and obliquely incident angles. The receiving transducer can be ro-
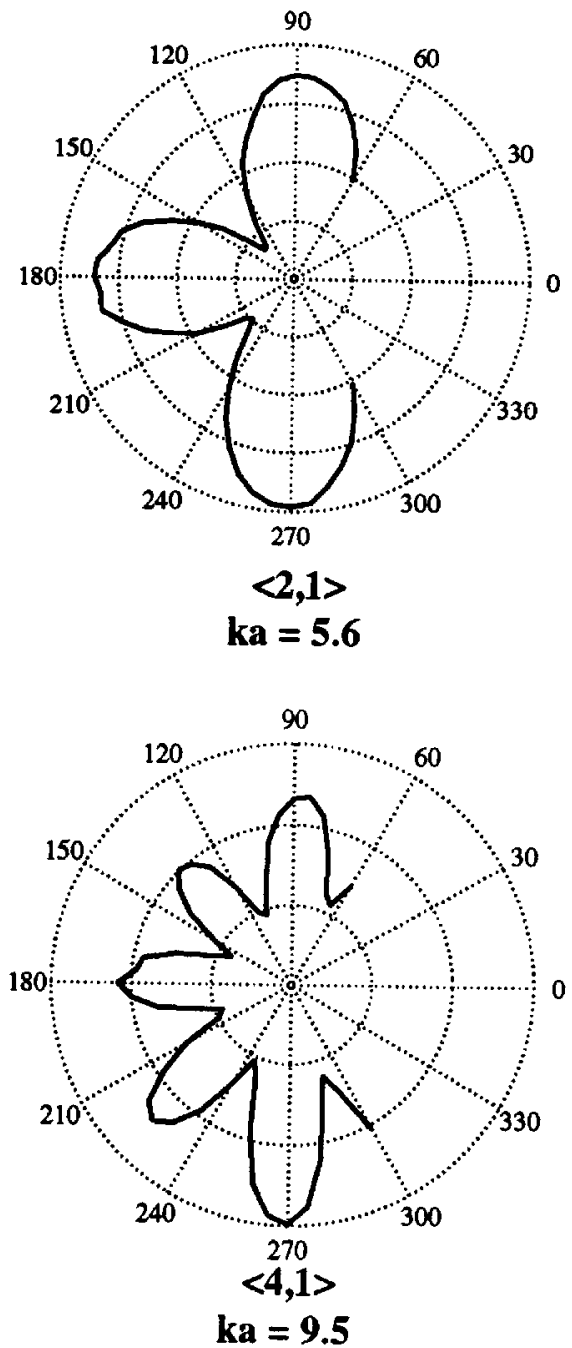

FIG. 12. Measured mode shapes of the copper-clad aluminum rod at incident angle of $\alpha=3^{\circ}$. 
tated in a horizontal plane several centimeters along the cylinder axis $z$ from that of the transmitting receiver. Thus, the resonances of the guided waves will be dominant in the acquired signal. In the measurements performed, the vertical distance (along the $z$ axis) between the transmitter and the receiver was about $10 \mathrm{~cm}$. The receiving transducer was swept through angles from $60^{\circ}$ to $300^{\circ}$ at $5^{\circ}$ increments. Because of the symmetry of the cylinder, sweeping the receiving transducer through a range of at least $180^{\circ}$ is sufficient for verification of the exact mode shape. ${ }^{28}$ Figure 12 shows samples of the measured mode shapes of whispering gallery-type waves and guided waves for an incident angle of $\alpha=3^{\circ}$.

Measurement of the mode shape of a resonance is a practical means for identifying the mode number of that resonance. The mode number is simply half the number of nodes or antinodes around the cylinder.

\section{CONCLUSIONS}

The problem of the scattering of an obliquely incident plane acoustic wave from a two-layered solid elastic cylinder (clad rod) has been formulated. Using RST, the dependence of resonance frequencies on the thickness of the cladding was evaluated. It is shown that different resonances react differently to the variations of the cladding thickness. Their sensitivity to these variations depends on the thickness of the cladding. In general, high frequency resonances are more sensitive than low frequency ones. Therefore, for NDE purposes, appropriate resonances can be selected to monitor or detect variations in cladding thickness.

The scattered pressure field from a copper-clad aluminum rod was measured using short pulses. The experimental measurements were consistent with numerical calculations and clearly showed the presence of all the predicted resonances.

The results explain one of the discrepancies between calculated and experimental diffraction spectra encountered in past studies of cylindrical components: At a nominal angle of incidence $\alpha=0^{\circ}$, the oblique components of the ultrasonic beam originating from non-planar characteristics of the wave generated by a finite-diameter transducer produce traces of guided-mode resonances not predicted by theory.

Using short pulse MIIR, the mode shapes of different resonances were measured experimentally for $\alpha=3^{\circ}$.

\section{ACKNOWLEDGMENTS}

This work was supported by a grant from the Manufacturing Research Corporation of Ontario (MRCO), and the National Science and Engineering Research Council (NSERC) of Canada. One of the authors (FH) acknowledges the financial support of the Ministry of Culture and Higher Education of Iran.

${ }^{1}$ H. Überall, "Surface Waves in Acoustics," in Physical Acoustics (Academic, New York, 1973), Vol. 10, Chap. 1

${ }^{2}$ W. Franz, Z. Naturforsch. 9a, 705 (1954).

${ }^{3}$ G. V. Frisk, J. W. Dickey, and H. Überall, J. Acoust. Soc. Am. 58, 996 (1975).

${ }^{4}$ J. W. Dickey, G. V. Frisk, and H. Überall, J. Acoust. Soc. Am. 59, 1339 (1976).

${ }^{5}$ R. H. Vogt and W. G. Neubauer, J. Acoust. Soc. Am. 60, 15 (1976).

${ }^{6}$ G. Maze, J. L. Izbicki, and J. Ripoche, J. Acoust. Soc. Am. 77, 1352 (1985)

${ }^{7}$ J. L. Izbicki, G. Maze, and J. Ripoche, J. Acoust. Soc. Am. 80, 1215 (1986)

${ }^{8}$ F. Léon, F. Lecroq, D. Décultot, and G. Maze, J. Acoust. Soc. Am. 91, 1388 (1992).

${ }^{9}$ A. Nagl, H. Überall, P. P. Delsanto, J. D. Almar, and E. Rosario, Wave Motion 5, 235 (1983).

${ }^{10}$ J. J. Faran, Jr., J. Acoust. Soc. Am. 23, 405 (1951).

${ }^{11}$ L. Flax and W. G. Neubauer, J. Acoust. Soc. Am. 63, 675 (1978).

${ }^{12}$ L. Flax, V. K. Varadan, and V. V. Varadan, J. Acoust. Soc. Am. 68, 1832 (1980).

${ }^{13}$ N. D. Veksler, Acustica 71, 111 (1990).

${ }^{14}$ J. M. Conoir, P. Rembert, O. Lenoir, and J. L. Izbicki, J. Acoust. Soc. Am. 93, 1300 (1993).

${ }^{15}$ G. Maze, B. Taconet, and J. Ripoche, Phys. Lett. 84A, 309 (1981).

${ }^{16}$ M. de Billy, J. Acoust. Soc. Am. 79, 219 (1986).

${ }^{17}$ G. Quentin and A. Cand, Electron. Lett. 25, 353 (1989).

${ }^{18}$ L. Flax and W. G. Neubauer, J. Acoust. Soc. Am. 61, 307 (1977).

${ }^{19}$ A. N. Sinclair and R. C. Addison, Jr., J. Acoust. Soc. Am. 94, 1126 (1993).

${ }^{20}$ J. Sinai and R. C. Waag, J. Acoust. Soc. Am. 83, 1728 (1988).

${ }^{21}$ J. A. Roumeliotis and N. B. Kakoginnos, J. Acoust. Soc. Am. 97, 2074 (1995).

${ }^{22} \mathrm{~F}$. Honarvar, "Nondestructive evaluation of cylindrical components by resonance acoustic spectroscopy," $\mathrm{PhD}$ thesis, University of Toronto, 1997.

${ }^{23}$ L. Flax, G. C. Gaunaurd, and H. Überall, "Theory of Resonance Scattering," in Vol. 15 of Physical Acoustics (Academic, New York, 1981), Chap. 3, pp. 191-294.

${ }^{24}$ H. D. Dardy, L. Flax, and C. F. Gaumond, J. Acoust. Soc. Am. 82, 1378 (1987).

${ }^{25}$ A. Briggs, Acoustic Microscopy (Oxford, New York, 1992).

${ }^{26} \mathrm{~T}$. Li and M. Ueda, J. Acoust. Soc. Am. 86, 2363 (1989).

${ }^{27}$ J. Krautkrämer and H. Krautkrämer, Ultrasonic Testing of Materials (Springer Verlag, New York, 1983), 3rd ed.

${ }^{28}$ H. Überall, Acoustic Resonance Scattering (Gordon and Breach Science, Philadelphia, 1992). 